\title{
TUBERCULOSIS IN A NIGERIAN MEDIUM SECURITY PRISON
}

\author{
${ }^{*}$ M. A. Lawal, **M. Omili, *T. O. Bello, *L. Onuha, *A. Haruna. \\ *Medical unit, Kuje Medium Security Prison, Kuje, Abuja, Nigeria, ${ }^{* *}$ Prison Medical \\ Centre, Prison Village, Airport Road, Abuja, Nigeria.
}

\section{Correspondence:}

Dr M. Omili

Prison Medical Centre

Prison Village, Airport Road,

Abuja, Nigeria.

Email: perryrx@yahoo.com

\begin{abstract}
Introduction: Prisons worldwide have been cited as reservoirs for tuberculosis and also an ideal setting for interventions but little is known about the epidemiology and control of tuberculosis in Nigerian Prisons.
\end{abstract}

Objective: To determine the prevalence of tuberculosis, role of prison in its control and the mortality from tuberculosis among prison inmate in Kuje prison, Abuja, Nigeria.

Materials and Methods: This study was a retrospective study of cases of tuberculosis in Kuje prison between April, 2004 and December, 2008. Data was collected from their medical records. The inclusion criterion was: (1) Cases of tuberculosis in which the diagnosis was based on clinical history, smear positive test and chest $x$-ray radiograph. (2) Cases where the treatment of tuberculosis was according to treatment strategy recommended by World Health Organization.

Results: A total of forty-eight inmates, aged 20 to 45years, were diagnosed with tuberculosis within the period of interest. The prevalence rate was $2.4 \%$, based on a total prison population of 2002. Majority of cases $93.8 \%$ were first detected in Prison and the remaining $6.3 \%$ were detected prior to incarceration. Out of the $6.3 \%$ diagnosed prior to incarceration, $2.1 \%$ and $4.2 \%$ of cases have defaulted from DOTS for 2 weeks and more than 4 weeks respectively.

Majority $(66.7 \%)$ completed the treatment before discharging from prison, $29.2 \%$ were discharged from prison at various level of treatment and $4.2 \%$ of cases died while on treatment. Those who died were co-infected with HIV

Conclusion: It was evident from this study that: The prevalence of tuberculosis in Kuje prison was high, prison has a role in diagnosis and treatment, adherence and treatment outcome were good and the main indicator for mortality was HIV infection.

Primary prevention measures specifically targeting prisoners are needed to reduce prevalence of tuberculosis and also to achieve the objectives of National Health Policy on tuberculosis and leprosy.

Keywords: Prison, tuberculosis, control, mortality

\section{INTRODUCTION}

Tuberculosis (TB) is an infectious disease caused by mainly Mycobacterium tuberculosis in humans $^{1}$. Other mycobacteria such as Mycobacterium bovis, Mycobacterium africanum, Mycobacterium canetti, and Mycobacterium microti are significant cause of TB in some parts of Africa ${ }^{2,3}$. 
Tuberculosis is the most frequently occurring infectious disease in the world and also stands out as a major cause of morbidity, disability and death globally ${ }^{4}$. It accounts for 2-3 million deaths per annum, globally ${ }^{4,5}$. In 2006, a total of 1.7 million people died of TB including 231,000 people with $\mathrm{HIV}^{6}$. One-third of the world's current population has been infected with $M$. tuberculosis, and new infections occur at a rate of one per second ${ }^{4-6,7}$. Approximately $95 \%$ of new cases and $98 \%$ of deaths occur in developing nations. This is probably due limited resources availability and higher human immunodeficiency virus (HIV) infections ${ }^{5}$. The annual incidence rate is 356 per 100,000 cases in Africa ${ }^{6}$.

In 1993, the World Health Organization (WHO) declared TB as a global emergency. This is first disease so classified in the history of that organization and the Stop TB Partnership developed a Global Plan to Stop Tuberculosis with the aim of saving 14 million lives between 2006 and $2015^{8}$. TB is one of the few diseases for which specific control targets have been set in the Millennium Development Goals ${ }^{9}$. It directly causes an annual decline in workers productivity to the tune of US $\$ 13$ billion. TB is contagious and spreads through the air. If untreated, each person with active TB infects on average of 10 to 15 people every year ${ }^{6}$. The probability of transmission from one person to another depends upon the number of infectious droplets expelled by a carrier, the effectiveness of ventilation, the duration of exposure, and the virulence of the $M$. tuberculosis strain ${ }^{10}$.

Prisoners population represent a high proportion of poorly educated and socioeconomically disadvantaged who have increased risk of ill health, including a high risk of tuberculosis infection and disease. Other conditions that increase the risk include intravenous drug abuse; overcrowding, poor nutrition, poor hygiene, and unguaranteed access to improved prison health services. The prison population has a high burden of disease may be because access to medical care is limited and the population is made of substance abusers, homeless, mentally ill, ethnic minorities, asylum seekers, immigrants, people who inject drugs using unsanitary needles, medically under-served and low-income populations $^{11-19}$.

In tuberculosis control, health workers' caliber and adequacy largely determine the program quality and efficiency, as workers consume the bulk of running costs and manage the other resources. The World Health Organization (WHO) Global Plan to Stop TB 2006 - 2015 acknowledges that the main human resource issues affecting tuberculosis control are insufficient quality, quantity and distribution of health workers ${ }^{20}$.

Tuberculosis is diagnosed definitively by identifying the causative organism in a clinical sample like sputum or pus. When this is not possible, a probable diagnosis may be made using imaging, commonly chest X-rays and/or a tuberculin skin test. The DOTS (Directly Observed Treatment Shortcourse) is now showing promising results in effectively treating all TB in the community. This is strategy of tuberculosis treatment based on clinical trials done in the 1970s by Tuberculosis Research Centre, Chennai, India, focusing on a neglected area of infectious disease control is now showing promising results in effectively treating all TB cases in the community.

There is dearth of information about TB in the Nigerian prisons and Nigeria 
being said to have the world's fourth largest tuberculosis (TB) burden, with more than 460,000 estimated new cases in $2007^{21}$.

The objective of this study was to determine the prevalence of tuberculosis, role of prison in its control and the mortality from tuberculosis among prison inmate in Kuje prison, Abuja, Nigeria.

\section{MATERIALS AND METHODS}

This was a retrospective study of cases of Tuberculosis in Kuje Prison between April 2004 and December 2008. The inclusion criteria include:

1. Cases of tuberculosis in which the diagnosis was made on clinical history, smear positive test and chest radiograph.

2. Cases of TB where treatment of tuberculosis was according to the treatment strategy recommended by World health organization (i.e DOTS).

\section{RESULTS}

A total of number of inmates recorded over the study period was 2002 with awaiting trial as $1654(82.6 \%)$ and convict as $348(17.4 \%)$. the ratio of convict to awaiting trial inmates was 1:4.8. The population of inmates increased steadily over the study period except in 2008 when a decline was seen (Table I). A total of fortyeight inmates, aged 20 to 45years, were diagnosed with tuberculosis within the period of interest. There were all awaiting trial inmates. The prevalence rate was $2.4 \%$.

Table I: NO OF INMATES WITHIN STUDY PERIOD

\begin{tabular}{llll} 
Year & Awaiting Trial (\%) & Convicted (\%) & Total (\%) \\
\hline 2004 & $258(15.6)$ & $42(12.1)$ & $300(15.0)$ \\
2005 & $215(13.0)$ & $65(18.7)$ & $280(14.0)$ \\
2006 & $327(19.8)$ & $71(20.4)$ & $398(19.9)$ \\
2007 & $453(27.4)$ & $88(25.3)$ & $541(27.0)$ \\
2008 & $401(24.2)$ & $82(23.6)$ & $483(24.1)$ \\
\hline Total & $\mathbf{1 6 5 4 ( 1 0 0 )}$ & $\mathbf{3 4 8 ( 1 0 0 )}$ & $\mathbf{2 0 0 2 ( 1 0 0 )}$
\end{tabular}


Fig. I: PLACE OF TUBERCULOSIS DIAGNOSIS

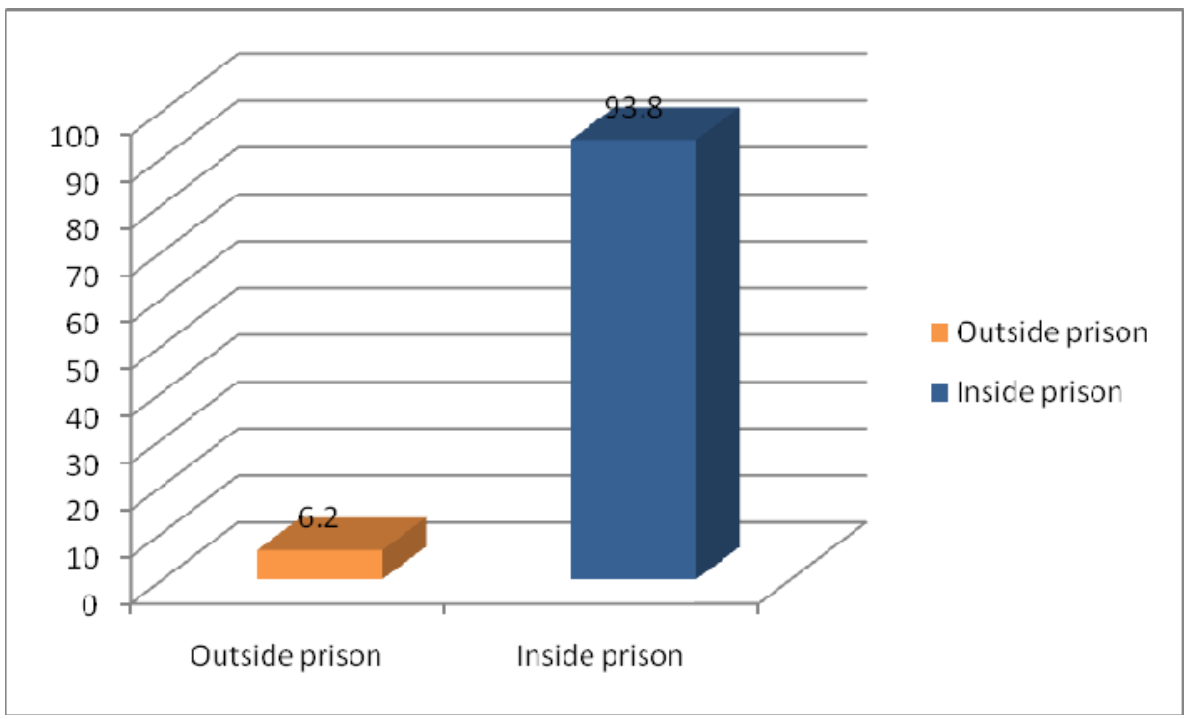

Majority of cases $93.8 \%$ were first detected in Prison and the remaining $6.3 \%$ were detected prior to incarceration (Fig. I). Out of the $6.3 \%$ diagnosed prior to incarceration, $2.1 \%$ and $4.2 \%$ of cases have defaulted from DOTS for 2 weeks and more than 4 weeks respectively.

\section{Fig. II: OUTCOME OF TREATMENT}

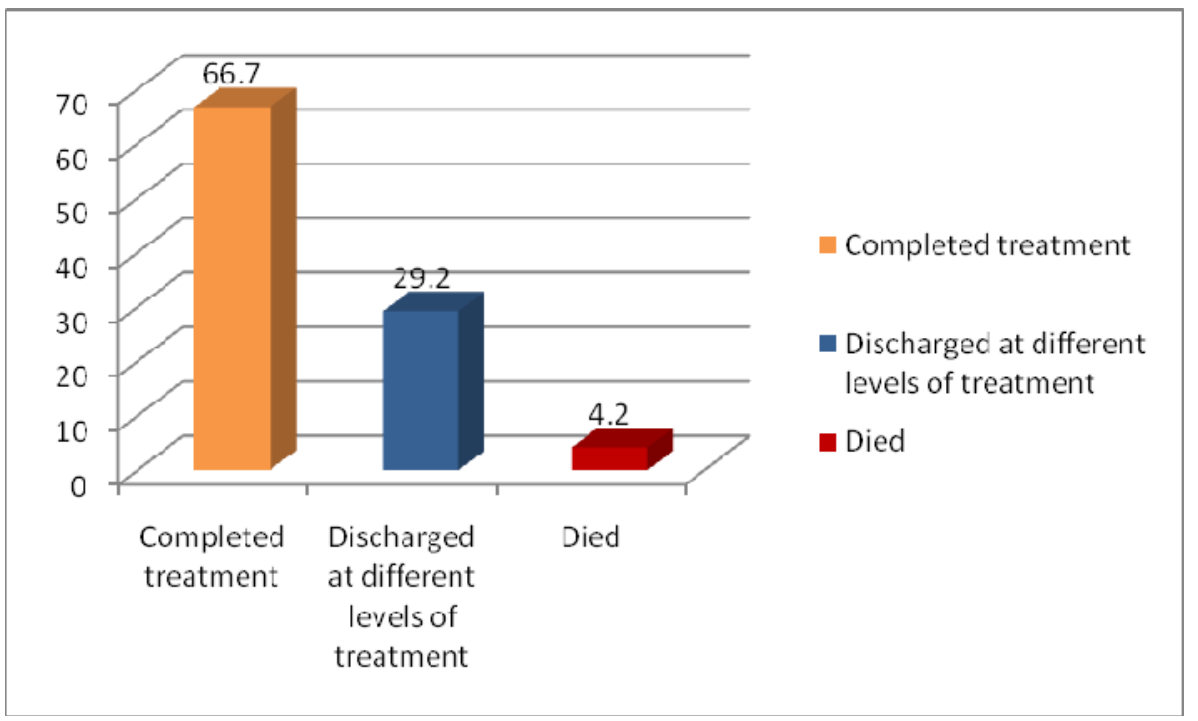

Majority $66.7 \%$ completed the treatment before discharging from prison, $29.2 \%$ were discharged from prison at various level of treatment and
$4.2 \%$ of cases died while on treatment (Fig. II). Those that were co-infected with HIV died in the course of the treatment (Fig. III). 
Fig. III: CO-INFECTION WITH HIV

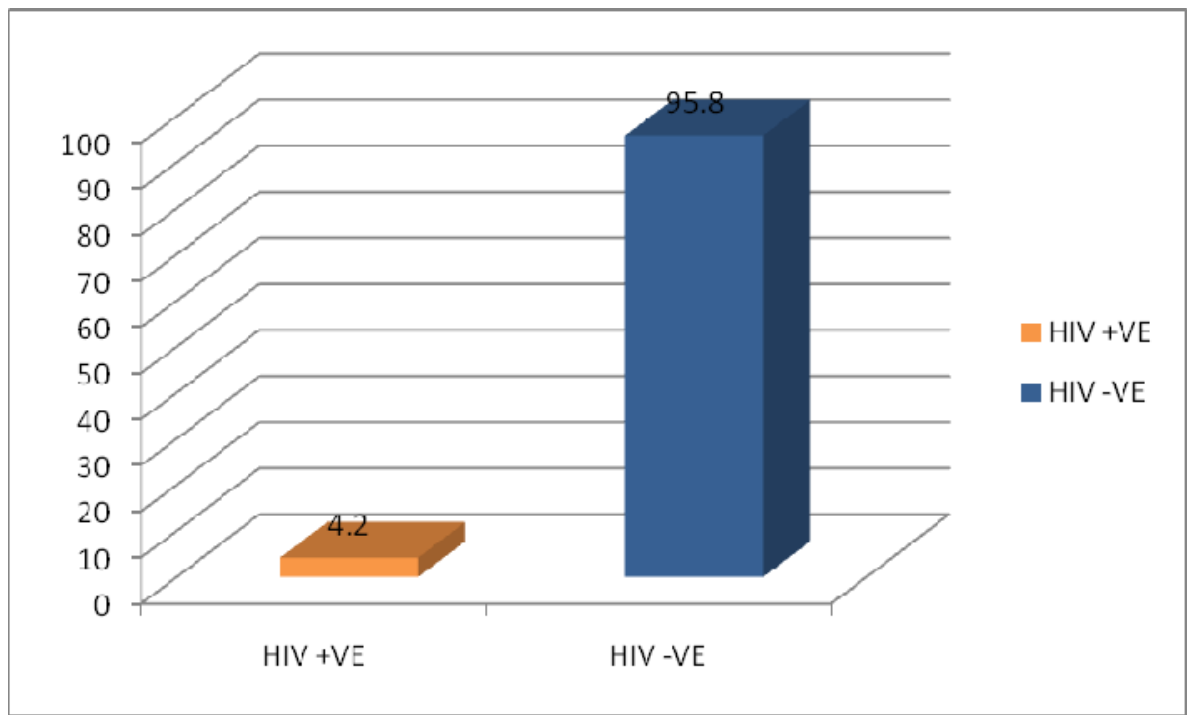

\section{DISCUSSION}

TB occurs predominantly in resourcelimited countries and represents a substantial public health problem in low- and middle-income countries. The World Health Organization and the International Committee of the Red Cross have joined forces to produce guidelines for the control of tuberculosis in prisons and similar institutions in countries with a high prevalence of tuberculosis. The internationally recommended strategy for tuberculosis control relies on early detection and cure of patients, with a priority for infectious cases. Screening of prisoners on entry into prison may have a role in early case detection. This is reflected by the study as $10 \%$ in this study were already being diagnosed with tuberculosis before incarceration. Overcrowding, poor nutrition, poor hygiene and long prison sentences promote tuberculosis. These are dominant features of Nigeria prisons and this may explain why $90 \%$ of cases were first diagnosed in prison. Overcrowding, poor ventilation, poor nutrition and inadequate or inaccessible medical care can facilitate the spread of disease in the prisons.
Prisons act as a reservoir for TB, pumping the disease into the civilian community through staff, visitors and inadequately treated former inmates ${ }^{22}$. Therefore, improving TB control in prisons will benefit the community at large. Community TB control efforts cannot afford to ignore prison TB.

Most prisoners come from underprivileged sectors of the general population and are more likely to have contracted tuberculosis before their arrival in prison.

The prevalence of $2.4 \%$ recorded in this study is lower than $4.0 \%$ recorded in Zambian prisons $^{23}$. All the cases of TB in this study were inmates of awaiting trial. This group poses a lot of risk as they come in contact with many people as they are moved from prisons to court rooms and when they receive visitors ranging from lawyers, human right groups and family members.

Certain features of the prison environment provide an opportunity to implement effective control programmes. For example, the captive audience in prisons should facilitate direct observation of treatment, complete coverage, and health education. In this present study, quite a 
high number (64\%) completed their treatment of tuberculosis while still in prison and $32 \%$ discharged at different level of treatment were adequately motivated to complete the treatment outside the prison in DOTS near their residence.

Judicial services are slow and this has been condemned in many quarters. This long stay before trial served as an opportunity to achieve complete treatment of tuberculosis in two third $(66.7 \%)$ of the affected group. Long duration of antituberculosis negatively impacts on adherence to therapy. In this study, previously diagnosed inmates before incarceration defaulted. Confinements removed cases of defaulting which is a positive side of imprisonment. This substantiated the fact that a prison is a control setting for TB control ${ }^{24,25}$.

Tuberculosis is a leading cause worldwide of morbidity and mortality among HIV-infected people ${ }^{26}$. In this study, the mortality rate was $4.2 \%$. The co-infection with HIV infection was the predictor of mortality. Prison conditions, tuberculosis, and HIV transmission are interconnected. Prevalence of HIV is higher in prison than the society due Illegal drug use, Sex between men, voluntary or forced, and rare use of condoms $^{27,28}$. The HIV epidemic complicates control of tuberculosis in prisons. This is confirmed in this study as $4.2 \%$ of cases were HIV-infected and were the only ones that died during treatment.

\section{CONCLUSION}

It was evident from the study that the prison has a role in the diagnosis and treatment of TB. Mortality was low and the main indicator was HIV infection. Adherence and treatment outcome were good. Emphasis should be placed on primary prevention to reduce the prevalence of tuberculosis in prison. It is also recommended that prisons should be well-ventilated, overcrowding should be prevented and good nutrition provided to reduce the tuberculosis prevalence to the barest minimum inorder to achieve the objectives of National health policy on tuberculosis and leprosy.

\section{REFERENCES}

1. Kumar, Vinay; Abbas, Abul K.; Fausto, Nelson; \& Mitchell, Richard N. Robbins Basic Pathology (8th ed.). Saunders Elsevier. 2007 pp. 516-522.

2. Niemann $S$, Rüsch-Gerdes $S$, Joloba $\mathrm{ML}$, et al Mycobacterium africanum subtype II is associated with two distinct genotypes and is a major cause of human tuberculosis in Kampala, Uganda. J. Clin. Microbiol. 2002; 40(9): 3398-405.

3. Niobe-Eyangoh SN, Kuaban C, Sorlin P, et al. Genetic biodiversity of Mycobacterium tuberculosis complex strains from patients with pulmonary tuberculosis in Cameroon. J. Clin. Microbiol. 2003; 41(6): 2547-53.

4. Tomioka H, Namba K. Development of antituberculous drugs: current status and future prospects. Kekkaku. 2006; 81(12):753-74.

5. Ducati RG, Ruffino-Netto A, Basso LA, Santos DS. The resumption of consumption -- a review on tuberculosis. Mem Inst Oswaldo Cruz. 2006; 101(7):697-714.

6. World Health Organization (WHO). Tuberculosis Fact sheet $\mathrm{N}^{\circ} 104$ Global and regional incidence. March 2006, Retrieved on 6 October 2006.

7. Centers for Disease Control and Prevention (CDC).Plan to combat 
extensively drug-resistant tuberculosis: recommendations of the Federal Tuberculosis Task Force. MMWR Recomm Rep. 2009; 58(RR-3):1-43.

8. World Health Organization (WHO). Stop TB Partnership. Retrieved on 3 October 2006.

9. United Nations. End poverty 2015. http://www.un.org/millenniumgoals/

10.Centers for Disease Control and Prevention (CDC), Division of Tuberculosis Elimination. Core Curriculum on Tuberculosis: What the Clinician Should Know. 4th edition (2000). Updated August 2003.

11. Griffith D, Kerr C. Tuberculosis: disease of the past, disease of the present. J Perianesth Nurs 1996; 11(4): 240-5.

12.Zolopa AR, Hahn JA, Gorter R, Miranda J, Wlodarczyk D, Peterson J, Pilote L, Moss A. HIV and tuberculosis infection in San Francisco's homeless adults: prevalence and risk factors in a representative sample. J Am Med Assoc. 1994; 272: 455-461.

13. Centers for Disease Control and Prevention. Screening for tuberculosis and tuberculosis infection in high-risk populations. Recommendations of the Advisory Council for the Elimination of Tuberculosis. Morbidity and Mortality Weekly Report. 1995; 44(RR-11):19-34.

14. Barnes PF, El-Hajj $H$, PrestonMartin S, Cave MD, Jones BE, Otaya M. Pogoda J, Eisenach, KD. Transmission of tuberculosis among the urban homeless. Journal of the
American Medical Association. 1996; 275:305-307.

15. Harlow CW. Profile of Jail Inmates 1996. US Department of Justice, Office of Justice Programs, Bureau of Justice Statistics, Washington, DC. 1998.

16. Bureau of Justice Statistics. HIV in Prisons and Jails, 1993. NCJ152765. US Department of Justice, Office of Justice Programs, Bureau of Justice Statistics, Washington, DC. 1995.

17. Glaser J, Greifinger R. Correctional health care: a public health opportunity. Annals of Internal Medicine. 1993; 118:139-145.

18. Pelletier AR, DiFerdinando GT, Greenberg AJ, Sosin DM, Jones, W, Block A, Woodley CL. Tuberculosis in a correctional facility. Archives of Internal Medicine. 1993; 153:26922695.

19. Hammett T, Harrold L. Tuberculosis in Correctional Facilities, Issues and Practices. A Collaboration between the National Institute of Justice and the Centers for Disease Control and Prevention. US Department of Justice, Washington, DC. 1994.

20.World Health Organization. The global plan to stop TB 2006 - 2015. Stop TB Partnership, Geneva, WHO; 2006.

21. USAID health: Infectious diseases, Tuberculosis Countries, Nigeria. http://www1.usaid.gov/our work/glo bal health/id/tuberculosis/countries/ africa/nigeria profile.html

22. Aerts A, Hauer B, Wanlin M, Veen J. Tuberculosis and tuberculosis control in European prisons. Int $\mathrm{J}$ 
Tuberc Lung Dis. 2006; 10(11):1215-23.

23. Habeenzu C, Mitarai S, Lubasi D, Mudenda V, Kantenga T, Mwansa J, Maslow JN. Tuberculosis and multidrug resistance in Zambian prisons, 2000-2001. Int J Tuberc Lung Dis. 2007; 11(11):1216-20.

24. Coninx $R$, Maher $D$, Reyes $H$, Grzemska M. Tuberculosis in prisons in countries with high prevalence. BMJ 2000; 320: 440442.

\section{World Health Organization.} Tuberculosis control in prisons. A Manual for Programme Managers. WHO/CDS/TB/ 2000.281. Geneva, World Health Organization, 2000.

26. Mukadi YD, Maher D, Harries A. Tuberculosis case fatality rates in high HIV prevalence populations in sub-Saharan Africa. AIDS. 2001; 15(2):143-52.

27. Polonsky S, Kerr S, Harris B, Gaiter J, Fichtner RR, Kennedy MG. HIV prevention in prisons and jails: obstacles and opportunities. Public Health Rep. 1994; 109(5): 615-625.

28. Hammett TM. HIVIAIDS and other infectious diseases among correctional inmates: transmission, burden, and an appropriate response. Am J Public Health. 2006; 96(6):974-8. 